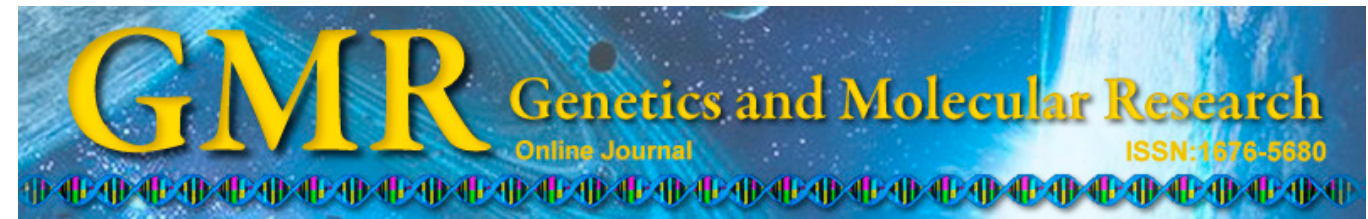

\title{
Construction of a lentiviral vector encoding heme oxygenase 1 and its introduction into mouse adipose tissue-derived stem cells
}

\author{
C.H. Zhu' ${ }^{1}$ W. Lei ${ }^{2}$ and Z.R. Chen ${ }^{1}$ \\ ${ }^{1}$ Department of Respiratory Medicine, \\ Children's Hospital of Soochow University, Suzhou, Jiangsu Province, China \\ ${ }^{2}$ Department of Respiratory Medicine, \\ First Affiliated Hospital of Soochow University, Suzhou, Jiangsu Province, China \\ Corresponding author: C.H. Zhu \\ E-mail: canhongzhu@sina.com
}

Genet. Mol. Res. 14 (3): 10705-10716 (2015)

Received December 9, 2014

Accepted May 29, 2015

Published September 9, 2015

DOI http://dx.doi.org/10.4238/2015.September.9.10

\begin{abstract}
Many studies exist concerning the use of stem cells as delivery vehicles in gene therapy, expressing genes such as vascular endothelial growth factor 165 and hepatocyte growth factor. However, few reports regarding adipose tissue-derived stem cells (ADSCs) and the heme oxygenase $1(H O-1)$ gene have been published. Therefore, we established a lentiviral vector encoding $\mathrm{HO}-1$ and used this to infect ADSCs with the aim of producing therapeutic seed cells. In this study, ADSCs were isolated from mouse adipose tissue (AT), cultured, and identified according to the expression of antigens on their cell surface and their capacity for multilineage differentiation. A lentiviral vector encoding $\mathrm{HO}-1$ was constructed, ADSCs were infected with this, and HO-1 protein expression was examined by western blotting. Our results show that ADSCs can be isolated from mouse AT, while DNA sequencing demonstrated that $\mathrm{HO}-1$ was successfully transferred to the vector fused with GFP. Following 293T cell transfection, lentivirus titers were approximately $3 \times 10^{8} \mathrm{TU} / \mathrm{mL}$.
\end{abstract}


Fluorescence microscopy confirmed the expression of the HO- 1 construct in lentivirus-infected ADSCs and the overexpression of HO-1 protein in these cells was verified by western blot. The production of ADSCs overexpressing HO-1 described in this study may aid in the development of a novel method for the treatment of asthma.

Key words: Adipose tissue-derived stem cells; Heme oxygenase 1; Lentivirus; Gene therapy; Asthma

\section{INTRODUCTION}

The definition of gene therapy is the permanent or transient alteration of an individual's genetic make-up, usually producing a therapeutic effect via the insertion or deletion of specific genes or sequences. Previously, gene therapy has predominantly focused on those illnesses for which no effective alternative therapies exist, such as cancer and cystic fibrosis. However, recent developments in this field have included work on other diseases, including asthma and allergic rhinitis (Maes et al., 2011).

Heme oxygenase (HO) is a rate-limiting enzyme that degrades heme into carbon monoxide, biliverdin, and ferritin (Tracz et al., 2007). In the airway, HO is expressed in various tissues and cells, including smooth muscle, epithelium, macrophages, endothelium, and parasympathetic ganglia (Lim et al., 2000). HO-1 is an inducible enzyme expressed in response to a number of stimuli, such as hydrogen peroxide, heat, heavy metal ions, superoxides, endotoxins, and inflammatory cytokines (Lee et al., 2010). Airway inflammation can be decreased by overexpression of HO-1, via the downregulation of tumor necrosis factor receptor 1-dependent oxidative stress and upregulation of foxp3 T-regulatory cells, interleukin-10, and membrane-bound transforming growth factor- $\beta 1$ (TGF- $\beta 1$; Xia et al., 2007; Lee et al., 2009). With this in mind, we attempted to package the $\mathrm{HO}-1$ gene into a lentiviral vector and use this for the treatment of asthma via the overexpression of HO-1 in the lungs. However, this lentiviral delivery method into lungs has proven to be problematic.

Mesenchymal stem cells (MSCs) are heterogeneous multipotent cells and can be isolated from many tissue types. A large number of studies have focused on MSCs obtained from bone marrow. However, numerous problems are associated with the clinical use of bone marrow MSCs, such as pain, morbidity, and the retrieval of low numbers of usable cells. In contrast, adipose tissue (AT) contains high numbers of easily isolated adipose tissue-derived stem cells (ADSCs), capable of differentiating into chondrogenic, osteogenic, endothelial, myogenic, and neurogenic lineages (Fraser et al., 2006; Liu et al., 2007). Animal studies have shown that ADSCs injected into the tail vein can target lung inflammation and display potential beneficial effects for asthma (Park et al., 2010).

ADSCs containing an $\mathrm{HO}-1$ gene construct could not only effect HO-1 overexpression in inflamed lung tissue, but in addition, convey their own anti-inflammatory properties. Thus, pulmonary ADSC transplantation combined with HO-1 expression may achieve significantly improved results over stem cell or gene therapy alone. In the present study, we cloned $H O-1$ complementary DNA (cDNA), generated a lentiviral vector encoding $\mathrm{HO}-1$, infected ADSCs with this vector, and examined the expression pattern of the HO-1 protein. Our study outlines a novel method for the treatment of asthma. 


\section{MATERIAL AND METHODS}

\section{Cloning of mouse $\mathrm{HO}-1 \mathrm{cDNA}$}

$\mathrm{BALB} / \mathrm{c}$ mice were sacrificed after having been anesthetized with $10 \%$ chloral hydrate. Excised spleens were rapidly trimmed, snap-frozen in liquid nitrogen, and stored at $-80^{\circ} \mathrm{C}$ until used for RNA extraction. Total RNA was isolated using the TRIzol (Invitrogen, Carlsbad, CA, USA) method, according to the manufacturer protocol. The integrity of RNA samples was checked by electrophoresis on an agarose gel stained with ethidium bromide. RNA concentration was determined by spectrophotometric absorbance at 260 and $280 \mathrm{~nm}$. Total RNA $(1 \mu \mathrm{g})$ was randomly reverse transcribed into cDNA by incubation at $42^{\circ} \mathrm{C}$ for 60 min with $1 \mu \mathrm{L}$ Moloney murine leukemia virus reverse transcriptase (Invitrogen), using $2 \mu \mathrm{L}$ oligo(dT) primers in a $5 \mathrm{X}$ reaction buffer containing $250 \mathrm{mM}$ Tris- $\mathrm{HCl}, \mathrm{pH} \mathrm{8.3,} 375$ $\mathrm{mM} \mathrm{KCl}, 15 \mathrm{mM} \mathrm{MgCl}, 50 \mathrm{mM}$ dithiothreitol, and $10 \mathrm{mM}$ dNTPs. Animal experiments were performed in accordance with the policies of the animal ethics committee of Soochow University.

\section{Construction of mouse $\mathrm{HO}-1$ expression vector}

The mouse $\mathrm{HO}-1$ coding region was amplified from mouse spleen cDNA, prepared as described above, by polymerase chain reaction (PCR) using the following pair of primers based on the conserved region of mouse HO-1 mRNA (GenBank accession No. NM_010442.2): Mus HmoX1-EcoRI-F, 5'-AAGAATTCATGGAGCGTCCACAGCCCGA-3', and Mus HmoX1BamHI-R, 5'-CGGGATCCTTACATGGCATAAATTCCCAC-3'. The resulting cDNA fragments were subcloned into a pMD18-T vector (pMD18-T-HO-1; Takara, Shiga, Japan). Then, pMD18-T-HO-1 and the pCDH-CMV-MCS-EF1-GFP-Puro vector (System Biosciences, Mountain View, CA, USA) were digested with BamHI and EcoRI (Takara) and linked to produce pCDH-CMV-MCS-EF1-GFP-HO-1. The HO-1 fragment within the pMD18-T and pCDH-CMV-MCS-EF1-GFP vectors was detected by electrophoresis on a $1 \%$ agarose gel. Positive clones were extracted and sequenced by Shanghai Genechem Co., Ltd. (Shanghai, China), and the final plasmid was named pCDH-CMV-HO-1.

\section{Preparation and titration of the lentiviral vector}

pCDH-CMV-HO-1 and two packaging components (psPAX2 and pMD2.G; Invitrogen) were extracted from positively transformed bacteria. The recombinant lentiviral vector, termed lenti-HO-1, was generated by cotransfecting $293 \mathrm{~T}$ cells with $20 \mu \mathrm{g}$ pCDHCMV-HO-1, $15 \mu \mathrm{g}$ psPAX2, and $6 \mu \mathrm{g}$ pMD2.G in a sterilized centrifuge tube containing Lipofectamine 2000 (Invitrogen). The 293T cells were cultured in L-Dulbecco's modified Eagle medium (L-DMEM; Invitrogen) containing 10\% fetal bovine serum (FBS; HyClone, GE Healthcare, Waukesha, WI, USA) in a $5 \% \mathrm{CO}_{2}$ incubator at $37^{\circ} \mathrm{C}$. Culture medium containing the lentivirus was harvested $48 \mathrm{~h}$ post-transfection and passed through a $0.45 \mu \mathrm{m}$ filter, before being concentrated twice by centrifugation at $4000 \mathrm{rpm}$ at $4^{\circ} \mathrm{C}$ for $10 \mathrm{~min}$. Virus aliquots were suspended in phosphate buffered saline (PBS) and stored at $-80^{\circ} \mathrm{C}$ until needed. Samples were diluted 1:1 before viral titers were measured. 


\section{Isolation, subculture, and identification of ADSCs}

\section{Isolation and subculture of ADSCs}

The procedure used in the current study to collect AT has been reported previously (Park et al., 2010). Briefly, AT was thoroughly washed with PBS, sliced into small pieces, and mixed with $0.075 \%$ collagenase I (Gibco, Carlsbad, CA, USA) to digest for $60 \mathrm{~min}$ at $37^{\circ} \mathrm{C}$. The resulting cells were washed twice with PBS and placed in three $50 \mathrm{~mL}$ tissue culture flasks at a density of $2 \times 10^{5}$ cells $/ \mathrm{mL}$, with L-DMEM medium containing $10 \%$ FBS being changed every 3 days. When the cells were more than $80 \%$ confluent, they were isolated and subcultured.

\section{Immunophenotype analysis of ADSCs}

Third passage ADSCs were used for immunophenotype analysis. Cells were resuspended in $100 \mu \mathrm{L}$ PBS at a concentration of $1 \times 10^{7}$ cells $/ \mathrm{mL}$ and incubated in the dark at $4^{\circ} \mathrm{C}$ for $30 \mathrm{~min}$ with fluorescein isothiocyanate (FITC)-conjugated rat anti-mouse CD90, CD44, CD11b, and CD45 antibodies (Becton Dickinson, Franklin Lakes, NJ, USA) at a concentration of $2 \mu \mathrm{g} / \mathrm{mL}$. As a negative control, cells were incubated with FITC-labeled rat anti-mouse IgG (Becton Dickinson). After being washed twice with PBS, the labeled cells were suspended in $500 \mu \mathrm{L}$ PBS. Then, fluorescence-activated cell sorting was performed using a FACSCalibur flow cytometer and analyzed with the CellQuest software (both Becton Dickinson).

\section{ADSC differentiation ability}

For adipogenic differentiation, third passage cells were incubated with L-DMEM medium containing $10 \%$ FBS supplemented with $500 \mu \mathrm{M}$ isobutylmethylxanthine, $1 \mu \mathrm{M}$ dexamethasone, $200 \mu \mathrm{M}$ indomethacin, $100 \mathrm{U} / \mathrm{mL}$ penicillin, $100 \mu \mathrm{g} / \mathrm{mL}$ streptomycin, and 0.01 $\mathrm{mg} / \mathrm{mL}$ insulin. After being cultured for 14 days, cells were fixed in $4 \%$ formalin, then incubated with oil red $\mathrm{O}$ for $15 \mathrm{~min}$ to visualize lipid droplets.

For osteogenic differentiation, third passage cells were incubated with L-DMEM medium containing $10 \%$ FBS supplemented with $0.1 \mu \mathrm{M}$ dexamethasone, $50 \mu \mathrm{g} / \mathrm{mL}$ ascorbic acid 2-phosphate, and $10 \mathrm{mM} \beta$-glycerophosphate disodium. After being cultured for 14 days, differentiation potential was assessed by modified Gomori and Von Kossa staining to detect alkaline phosphatase and calcium oxalate, markers of osteogenic differentiation.

For chondrogenic differentiation, third passage ADSCs were incubated in L-DMEM medium with $10 \%$ FBS supplemented with $10 \mu \mathrm{g} / \mathrm{L}$ TGF- $\beta 1,0.1 \mu \mathrm{M}$ dexamethasone, $1 \mathrm{mM}$ pyruvate, $6.25 \mu \mathrm{g} / \mathrm{mL}$ insulin, and $3 \mathrm{mg} / \mathrm{L}$ transferrin. After being cultured for 14 days, chondrogenic differentiation potential was assessed by Alcian blue staining.

\section{Infection of ADSCs by lentiviral vector}

Cells were seeded at a density of $1 \times 10^{5}$ cells $/ \mathrm{mL}$ on 24 -well plates in serum-free growth medium containing $8 \mu \mathrm{g} / \mathrm{mL}$ polybrene (SinoBio, Boston, MA, USA), with lenti-HO-1 at multiplicities of infection (MOI) of 50 and 100. Serum containing growth factors was added after $8 \mathrm{~h}$ and replaced after $48 \mathrm{~h}$. The expression of green fluorescent protein (GFP) was exam- 
ined using a fluorescence microscope $48 \mathrm{~h}$ later. The most appropriate MOI was determined, and subsequent infections of ADSCs were carried out according to this.

\section{Detection of HO-1 protein in ADSCs}

The extent to which the HO-1 protein was expressed in third passage ADSCs was assessed by western blot.Briefly, uninfected ADSCs, ADSCs infected with either lenti-HO-1 or lentivirus lacking the gene, and 293 cells containing the $\mathrm{HO}-1$ plasmid were harvested. One hundred microliter ice cold $2 \mathrm{X}$ lysis buffer was then added to the cells. The supernatant was collected, and the concentration of protein measured by bicinchoninic acid assay. Sample buffer (2X) was then applied to the samples (the volume of buffer added being determined by sample concentration), the proteins were separated by SDS-PAGE, and proteins were denatured at $100^{\circ} \mathrm{C}$ for $5 \mathrm{~min}$ before being transferred to polyvinylidene difluoride membranes at $20 \mathrm{~V}$ for approximately $1 \mathrm{~h}$. Subsequently, membranes were blocked with a solution of 5\% milk in Tris-buffered saline and Tween 20 (TBST), incubated with primary antibody (Mouse anti-HO-1 monoclonal antibody, 1:2000, Abcam, Cambridge, UK) at $4^{\circ} \mathrm{C}$ overnight, washed three times for 10 min with TBST, incubated with secondary antibody at room temperature for $1 \mathrm{~h}$, and washed 3 times again. Finally, enhanced chemiluminescence (ECL, Beyotime, Shanghai, China) reagent was applied to visualize protein bands. Films were exposed after having been incubated with ECL reagent for $5 \mathrm{~min}$, prior to being developed and fixed. Glyceraldehyde 3-phosphate dehydrogenase (anti-GAPDH, 1:2000, Abcam, Cambridge, UK) expression was used as a control to evaluate relative protein levels.

\section{RESULTS}

\section{Quality of mouse spleen total RNA}

The purity of total RNA samples was determined by spectrophotometric absorbance at 260 and $280 \mathrm{~nm}$. A260/A280 values were between 1.8 and 2.0, indicating very high RNA purity. The integrity of RNA was assessed visually on a non-denaturing gel. Distinct $28 \mathrm{~S}$ and $18 \mathrm{~S}$ ribosomal RNA bands were clearly observed, signifying high RNA integrity (Figure 1).

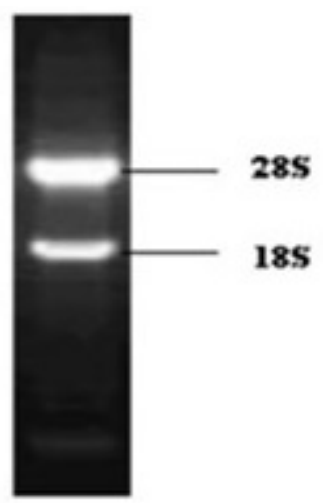

Figure 1. Agarose gel electrophoresis of total RNA. RNA was isolated from mouse spleen tissue using TRIzol reagent, then separated on a $1 \%$ agarose gel and stained with ethidium bromide. Ribosomal $28 \mathrm{~S}$ and $18 \mathrm{~S}$ bands are indicated and demonstrate the high integrity of the extracted RNA. 


\section{Vector construction and sequencing}

The entire HO-1 cDNA sequence was obtained by PCR amplification using specific primers and as predicted, the product of this reaction was approximately 870 bp (Figure 2). This was then cloned into the pMD18-T vector and the result assessed by electrophoresis on a $1 \%$ agarose gel (Figure 3). After linking pMD18-T-HO-1 and pCDH-CMV-MCS-EF1-GFPPuro following digestion with BamHI and EcoRI, the resulting pCDH-CMV-HO-1 plasmid was assessed by $1 \%$ agarose gel electrophoresis (Figure 4). In addition, the pCDH-CMVHO-1 vector was sequenced, confirming that the $\mathrm{HO}-1$ insert consisted of the same sequence as that listed in GenBank (accession No. NM_010442.2).

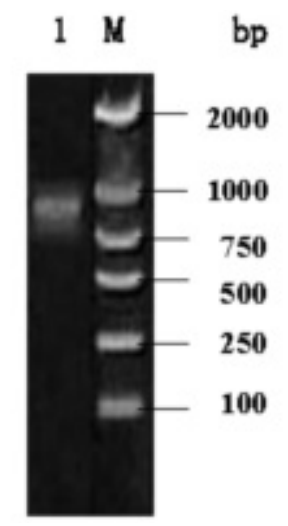

Figure 2. Agarose gel electrophoresis of complementary DNA (cDNA) synthesized from mouse spleen total RNA. cDNA was synthesized using reverse transcription polymerase chain reactions and separated on an ethidium bromide-stained agarose gel. Lane $M$ signifies the 2-kb DNA marker. Lane 1 shows the HO-1 fragment, which was slightly smaller than $1 \mathrm{~kb}$, consistent with the predicted $\mathrm{HO}-1$ amplicon size.

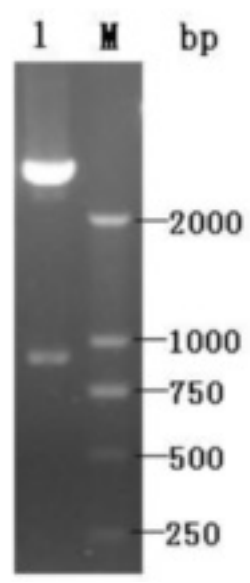

Figure 3. Agarose gel electrophoresis of the pMD18T-HO-1 vector. Lane $M$ signifies the 2-kb DNA marker. The upper band in lane 1 represents pMD18-T; the fragment was approximately $2.7 \mathrm{~kb}$, consistent with the plasmid's predicted size. The lower band represents $H O-1$, which was slightly smaller than $1 \mathrm{~kb}$, consistent with the size of the HO-1 insert. 


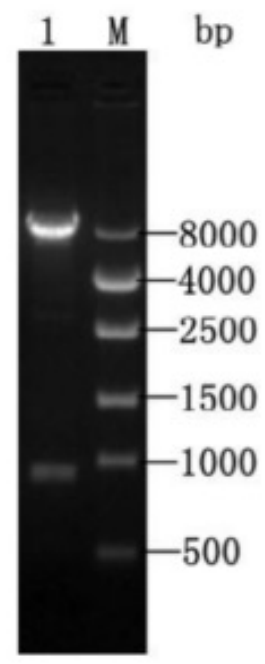

Figure 4. Agarose gel electrophoresis of the pCDH-CMV-HO-1 vector. Lane $M$ signifies the 8-kb DNA marker. The upper band in lane 1 represents pCDH-CMV-MCS-EF1-GFP-Puro; the fragment was larger than $8 \mathrm{~kb}$, consistent with the plasmid's predicted size. The lower band represents $H O-1$, which was slightly smaller than $1 \mathrm{~kb}$, consistent with the size of the HO-1 insert.

\section{Establishment and titration of the recombinant lentiviral vector}

After introducing the plasmids into 293T cells, GFP expression was evaluated using a fluorescence microscope to verify that the lentiviral vectors had been successfully generated (Figure 5). The viral titer was found to be $3 \times 10^{8} \mathrm{TU} / \mathrm{mL}$, after concentration.

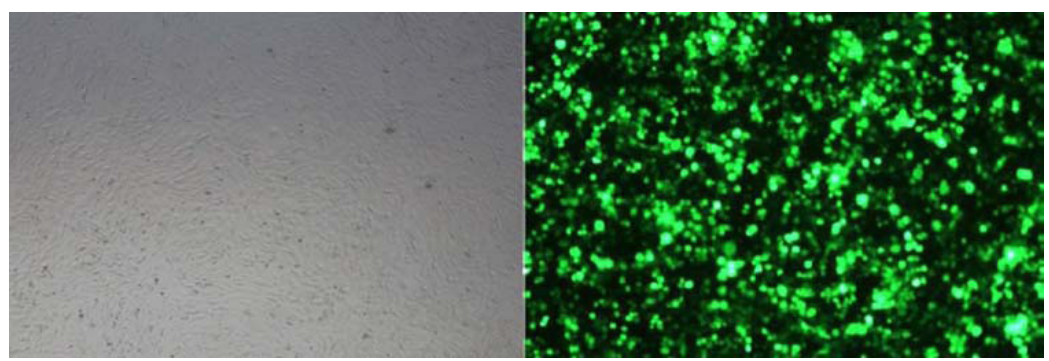

Figure 5. HO-1 recombinant lentiviral plasmid green fluorescent protein expression in $293 \mathrm{~T}$ cells $48 \mathrm{~h}$ after transfection (100X magnification). Left: bright field, right: dark field.

\section{Immunophenotype analysis of ADSCs}

To establish the ADSC surface antigen profile in more detail, we tested third passage cells for the expression of CD90, CD44, CD11b, and CD45. Flow cytometric analysis demonstrated that $96.6 \%$ of cells were positive for CD90 and $85.3 \%$ for CD44. Only $0.5 \%$ were positive for CD45 and CD11b (Figure 6). 

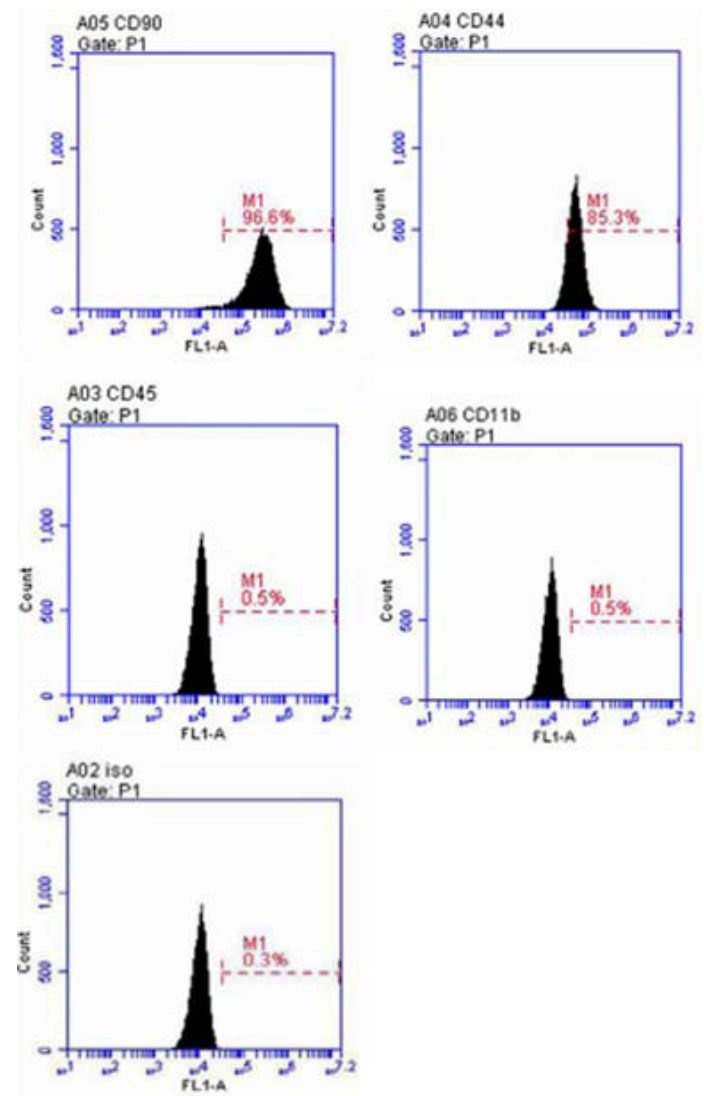

Figure 6. Expression of adipose tissue-derived stem cell (ADSC) surface markers. Flow cytometry histograms of third passage ADSCs for hematopoietic (CD11b and CD45) and stromal (CD90 and CD44) markers.

\section{Multilineage differentiation of ADSCs}

Oil red O staining revealed the presence of a small number of lipid droplets in the cytoplasm after ADSCs had been cultured in adipogenesis-inducing medium for 3 days. After 2 weeks, many more were observed and were seen to merge (Figure 7a).

Calcification was observed in ADSCs treated for 2 weeks in osteogenic medium, appearing as black particles within the cells when visualized with modified Gomori staining, and a black mineralized nodule when Von Kossa staining was employed (Figures $7 \mathrm{~b}$ and c).

Following 2 weeks in chondrogenic medium, several nodule-like structures were observed in the cytoplasm, as revealed by Alcian blue staining (Figure 7d).

\section{Infection efficiency of ADSCs with lentiviral vectors}

ADSCs were infected with lenti-HO-1 at an MOI of 50 or 100 and the expression of GFP was assessed using fluorescence microscopy (Figure 8). Fluorescence at an MOI of 100 was stronger than that at an MOI of 50 . 


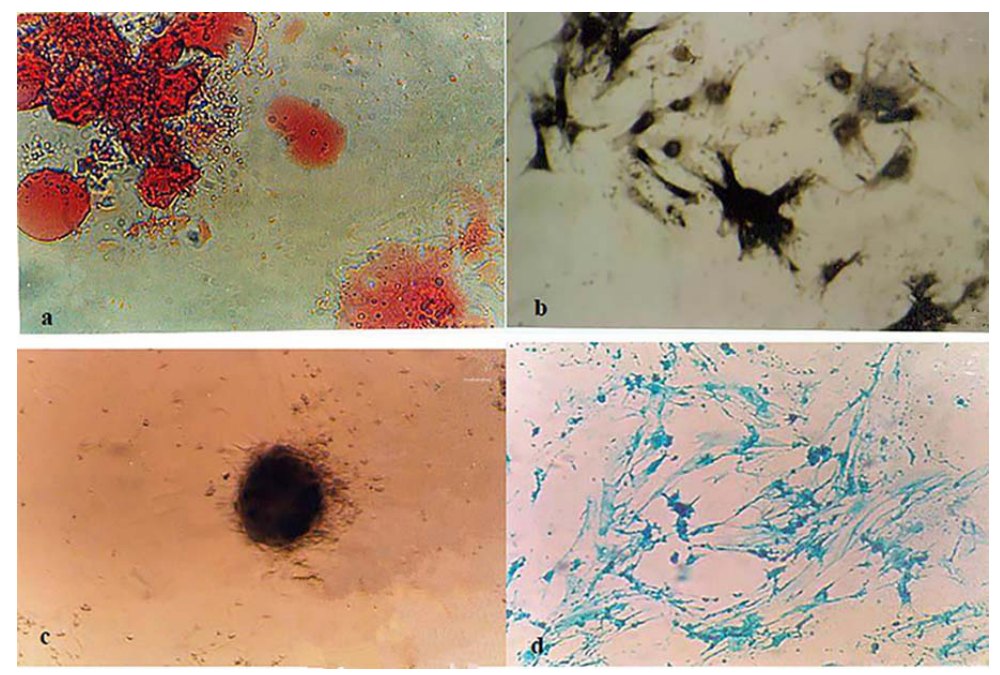

Figure 7. Plasticity of third passage adipose tissue-derived stem cells examined by differentiation of cells into particular lineages in the presence of appropriate induction media. (a) Cells showed the potential to differentiate into adipocytes, as assessed by oil red $\mathrm{O}$ staining; (b, c) modified Gomori and Von Kossa staining confirmed differentiation into osteoblasts; (d) Alcian blue staining confirmed differentiation into chondrocytes. Magnification: a, 400X; b, 200X; c and d, 100X.

a

b
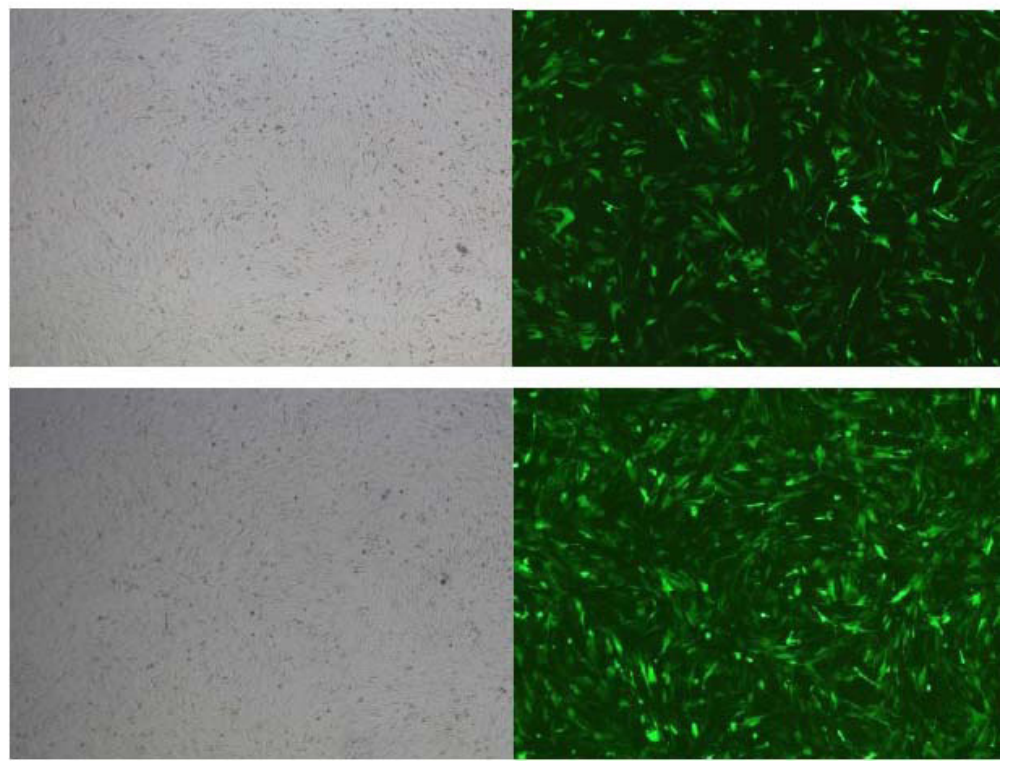

Figure 8. Infection of adipose tissue-derived stem cells with lenti-HO-1 at multiplicities of infection of 50 (upper panels) and 100 (lower panels). Expression of green fluorescent protein was observed via either light (a) or fluorescence (b) microscopy at 100X magnification. 


\section{Expression of HO-1 protein in ADSCs}

Neither non-infected ADSCs nor those infected with lentivirus lacking $\mathrm{HO}-1$ expressed HO-1 protein. A very high HO-1 expression level was observed in ADSCs infected with lenti-HO-1 and 293T cells containing the HO-1 plasmid (Figure 9).

1

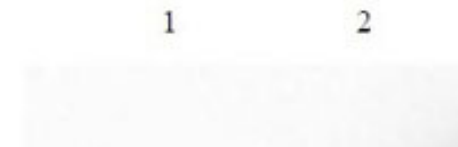

HO-1

34

GAPDH

Figure 9. Western blot analysis of HO-1 expression in mouse adipose tissue-derived stem cells (ADSCs) infected with lenti-HO-1. 1) Uninfected ADSCs as a negative control; 2) ADSCs infected with empty lentivirus; 3) ADSCs infected with lenti-HO-1; 4) $293 \mathrm{~T}$ cells containing the $\mathrm{HO}-1$ plasmid. HO-1 was visualized under a fluorescence microscope at 100X magnification. GAPDH was used as a loading control.

\section{DISCUSSION}

Asthma is a chronic airway inflammatory disease, and therapeutic strategies generally consist of attempts to control it using anti-inflammatory drugs, anti-leukotriene agents, and bronchodilators. Although these drugs can mitigate the symptoms of asthma and are beneficial for most patients, they do not constitute a cure. Therefore, it is necessary to develop safe, novel therapies that differ from the conventional approach, and are capable of providing a long-term treatment for allergic airway diseases.

It has been demonstrated that MSC therapy can be effective in treating asthma. Previous reports have indicated that MSCs injected into the tail vein decrease chronic airway inflammation, airway remodeling, and airway hyperreactivity in mice (Bonfield et al., 2010; Goodwin et al., 2011; Lee et al., 2011b). However, stem cell therapy in itself is associated with some difficulties, for example, stem cells are unstable and their numbers are low, as is their activity. Therefore, the administration of stem cells together with specific cytokines is likely to be of greater benefit, and this combination could provide a novel therapy for asthma.

Gene therapy is characterized by its capability for local treatment with long-lasting effects, made possible by its targeting important steps in the development of allergic diseases such as asthma and rhinitis. It can provide novel and effective therapeutic methods. Allergic airway diseases are polygenic disorders, as distinct from conditions such as cystic fibrosis, for which mutations in a single gene are almost wholly responsible. Environmental factors contribute considerably to the development of allergic diseases, and it is therefore unrealistic 
to target the "genetic predisposition" of asthma patients via gene therapy. However, future approaches will surely target specific mediators or cells that play an important function in the disease's pathogenesis to provide an alternative therapy for severe, uncontrolled asthma (Maes et al., 2011).

Recent studies have demonstrated that HO-1 plays numerous roles, involving antiinflammatory, anti-oxidant, anti-apoptotic, and possibly immunomodulatory functions (Ryter et al., 2006). Almolki et al. (2004) reported that overexpression of $\mathrm{HO}$ has a notable protective effect and can decrease airway inflammation and hyperresponsiveness, mucus hypersecretion, and oxidative stress in a guinea pig model of allergic asthma. Lee et al. (2011a) reported that administration of Angelica dahurica extract can suppress allergic airway inflammation induced by ovalbumin and suggested that it may be employed as an anti-inflammatory drug for the treatment of asthma. The mechanism behind this effect involves upregulation of HO-1. In the current study, we constructed an HO-1 expression vector and confirmed, using BLAST, that the HO- 1 insert sequence matched that of the corresponding transcript registered on GenBank (accession No. NM_010442.2; www.ncbi.nlm.nih.gov/BLAST/). As clinical application of HO-1 is significantly hindered by the short-term nature of its effects, gene therapy has been proposed as the optimal method for asthma treatment.

To express HO- 1 in the target cells, lentiviral vectors were chosen for their numerous beneficial properties, namely: i) lentiviral vectors can permanently deliver gene because vectors can stably integrate into host genome; ii) lentivirues not only infect dividing cells but also nondividing cells; iii) lentiviruses have broad tissue tropism and allow important types of gene- and cell-targeted therapy; iv) there is no expression of viral protein after vectors were transduced; v) lentiviruses have the ability to deliver complex genetic elements, such as polycistronic and intron-containing sequences; vi) lentiviruses have a potentially safer integration site profile; and vii) it is relatively easy to manipulate and product vectors. (Sakuma et al., 2012). We adopted a three-plasmid vector system and 293T cells for virus production. Viral titers reached $3 \times 10^{8} \mathrm{TU} / \mathrm{mL}$, which was sufficient for the infection of ADSCs.

In this study, we constructed a HO-1 recombinant lentiviral expression vector. The HO-1 plasmid was successfully established, and high virion titers were generated after plasmids were transfected into packaging cells using molecular cloning methods. Our western blot results showed that ADSCs containing the HO- 1 DNA construct overexpressed HO-1 protein. This demonstrated that the HO-1 gene had been successfully delivered into ADSCs by the lentiviral vector and that as a result, $\mathrm{HO}-1$ protein was highly expressed in these cells.

In future, ADSCs containing HO-1 constructs could be used to assess the efficacy of overexpressing this gene in combination with stem cell therapy in treating asthma and other airway diseases.

\section{Conflicts of interest}

The authors declare no conflict of interest.

\section{ACKNOWLEDGMENTS}

Research supported by grants from the Social Development Program of Suzhou City (\#SYS201250). 


\section{REFERENCES}

Almolki A, Taillé C, Martin GF, Jose PJ, et al. (2004). Heme oxygenase attenuates allergen-induced airway inflammation and hyperreactivity in guinea pigs. Am. J. Physiol. Lung Cell Mol. Physiol. 287: L26-L34.

Bonfield TL, Koloze M, Lennon DP, Zuchowski B, et al. (2010). Human mesenchymal stem cells suppress chronic airway inflammation in the murine ovalbumin asthma model. Am. J. Physiol. Lung Cell Mol. Physiol. 299: L760-L770.

Fraser JK, Wulur I, Alfonso Z and Hedrick MH (2006). Fat tissue: an underappreciated source of stem cells for biotechnology. Trends Biotechnol. 24: 150-154.

Goodwin M, Sueblinvong V, Eisenhauer P, Ziats NP, et al. (2011). Bone marrow-derived mesenchymal stromal cells inhibit Th2-mediated allergic airways inflammation in mice. Stem Cells 29: 1137-1148.

Lee IT, Luo SF, Lee CW, Wang SW, et al. (2009). Overexpression of HO-1 protects against TNF-alpha-mediated airway inflammation by down-regulation of TNFR1-dependent oxidative stress. Am. J. Pathol. 175: 519-532.

Lee NH, Lee MY, Lee JA, Jung DY, et al. (2010). Anti-asthmatic effect of Sanguisorba officinalis L. and potential role of heme oxygenase-1 in an ovalbumin-induced murine asthma model. Int. J.Mol. Med. 26: 201-208.

Lee MY, Seo CS, Lee JA, Lee NH, et al. (2011a). Anti-asthmatic effects of Angelica dahurica against ovalbumin-induced airway inflammation via upregulation of heme oxygenase-1. Food Chem. Toxicol. 49: 829-837.

Lee SH, Jang AS, Kwon JH, Park SK, et al. (2011b). Mesenchymal stem cell transfer suppresses airway remodeling in a toluene diisocyanate-induced murine asthma model. Allergy Asthma Immunol. Res. 3: 205-211.

Lim S, Groneberg D, Fischer A, Oates T, et al. (2000). Expression of heme oxygenase isoenzymes 1 and 2 in normal and asthmatic airways: effect of inhaled corticosteroids. Am. J. Respir. Crit. Care Med. 162: 1912-1918.

Liu TM, Martina M, Hutmacher DW, Hui JH, et al. (2007). Identification of common pathways mediating differentiation of bone marrow-and adipose tissue-derived human mesenchymal stem cells into three mesenchymal lineages. Stem Cells 25: 750-760.

Maes T, Tournoy KG and Joos GF (2011). Gene therapy for allergic airway diseases. Curr. Allergy Asthma Rep. 11: 163-172.

Park HK, Cho KS, Park HY, Shin DH, et al. (2010). Adipose-derived stromal cells inhibit allergic airway inflammation in mice. Stem Cells Dev. 19: 1811-1818.

Ryter SW, Alam J and Choi AM (2006). Heme oxygenase-1/carbon monoxide: from basic science to therapeutic applications. Physiol. Rev. 86: 583-650.

Sakuma T, Barry MA and Ikeda Y (2012). Lentiviral vectors: basic to translational. Biochem. J. 443: 603-618.

Tracz MJ, Alam J and Nath KA (2007). Physiology and pathophysiology of heme: implications for kidney disease. J. Am. Soc. Nephrol. 18: 414-420.

Xia ZW, Xu LQ, Zhong WW, Wei JJ, et al. (2007). Heme oxygenase-1 attenuates ovalbumin-induced airway inflammation by up-regulation of Foxp3 T-regulatory cells, interleukin-10, and membrane-bound transforming growth factor- $\beta 1$. Am. J. Pathol. 171: 1904-1914. 